\title{
New Anomaly at Low Temperature for Heat Capacity
}

\author{
Sonia Bouzgarrou1,2 \\ ${ }^{1}$ Microelectronic and Instrumentation Laboratory, Faculty of Sciences, Monastir University, Monastir, Tunisia \\ ${ }^{2}$ Department of Physics, College of Science, Qassim University, Buraydh, KSA \\ Email: b_sonia3@yahoo.fr
}

How to cite this paper: Bouzgarrou, S. (2020) New Anomaly at Low Temperature for Heat Capacity. Open Access Library Journal, 7: e6477.

https://doi.org/10.4236/oalib.1106477

Received: May 28, 2020

Accepted: July 28, 2020

Published: July 31, 2020

Copyright (c) 2020 by author(s) and Open Access Library Inc.

This work is licensed under the Creative Commons Attribution International License (CC BY 4.0).

http://creativecommons.org/licenses/by/4.0/

\section{(c) (i) Open Access}

\begin{abstract}
In this paper, we present a theoretical investigation as a function of temperature of a critical anomaly find in InAlAs hetero-structure of two-dimensional electron gas. This study has shown the presence of a large and continuous anomaly. This anomaly is explained through a theory based on the general assumption. The present theoretical research is based essentially on the characteristic of specific heat capacity extending over a large temperature, but we underline a good agreement with results of the relation with chemical potential and Broadening parameter as a function of temperature. It is found that the specific heat capacity observed by a peak at low temperature, at a critical temperature, is directly linked to Schottky anomaly and unveiling the existence of phase transition in InAlAs. Our results are completed by the study of the dependence of the heat capacity on the spin as a function of temperature. This study confirms the same behavior with result without spin.
\end{abstract}

\section{Subject Areas}

Applied Statistical Mathematics

\section{Keywords}

Two-Dimensional Electron Gas, Landeau Levels, Specific Heat Capacity, Anomaly, Critical Temperature, Chemical Potential, Broadening Parameter

\section{Introduction}

Electron in a Two-dimensional gas (2DEG) continues to be of interest in physics since they exhibit non classical behavior and are readily realizable in semiconductor hetero-structure [1] [2]. These last year's investigations on 2DEG (Twodimensional electron gas), are the object of many news research for example 
(Quantum Hall effect) [3] [4], and the Haas-van Alphen effect or the magnetization oscillations [5] [6] [7], and in the studies of the chemical potential [8] [9] [10] [11], and in the studies in the coupling between 2DEG and Spins [12] [13] [14] [15] [16].

On the side of theorical investigation, the general trend is attributed to the presence of Landeau levels. These Landeau levels are indicated by broadened energy levels, characterized by phenomenological broadening parameter $\Gamma$, which is represented in the density of states (DOS) [17] between eigenvalues $E_{n}$. These $E_{n}$, known as Landeau levels, are the quantized energy spectrum $(E)$ obtained when a strong electric field $(V)$ is applied to the system's plane [18] [19].

These striking behavior of $E_{n}$ broadening, from which other exotic features arises, has been attributed to disorder due to the presence of impurities, defects and other inhomogeneity's in the system [20] [21] [22]. These overlaps have been analytically confirmed in the presence of weak disorder [23]. But this derivation has no localization effect and contains parameters related to the impurity configuration, namely its density and distance from the 2DEG plane. Such conditions make quantitative comparison with experimental results difficult.

In our work, we present a numerical calculation of a new specific heat capacity $S_{\text {heat }}$ anomaly, characterized of InAlAs devices, for different chemical potential $\mu$, as a function of temperature $T$. The purpose of the present papers is to give furthers study for 2DEG. We show the presence of a large and continous anomaly at low temperature with the increase of chemical potential. Then we finish by a summary, that we have compared our results with other references.

\section{Numerical Model}

During the early part of the nineteenth century, studies on the heat capacity of materials tended to indicate that it was rather uninteresting property somewhat independent of temperatures. Heat capacity is one of the most fundamental physical properties as it directly probes thermodynamic quantities such as entropy [24] [25] [26] [27] [28]. In the case of two dimensional electrons gas (2DEGs) heat capacity can be a powerful probe of single and many body properties such as the Landau quantized density of states and the Quantum Hall effect (QHE). Both integral and fractional [29], measurement of the 2DEG heat capacity, however are among the most challenging experiments because of the very small electron contribution.

Thermodynamic properties of each device that can be described by the independent or free particle model are determined by their single particle energy spectra depending on the confinement and the size of the system.

For such system, the specific heat capacity of a substance $S_{\text {heat }}$ of 2 DEG was studied as a function of temperature $T$ [30] [31] [32]. At constant volume of an electron gas, heat capacity $S_{\text {heat }}$, in the Debye model [33] [34] [35], can be calculated directly from differentiation of the internal energy $U$, with respect to temperature [36] [37].

Specific heat capacity is one of the fundamental thermodynamic properties of 
a substance. It is defined as the energy that has to be transferred to or from a unit of mass or amount of substance to change the system temperature by one degree. The heat capacity of material is a property that indicates the amount of thermal energy the material must absorb to achieve a specified temperature rise. Specific heat capacity is generally sensitive to phase change. Different materials of a given mass require different quantities of heat to rise their temperature by specified value since different materials absorb energy in different ways. Specified heat capacity of material is obtained from expression:

$$
S_{\text {heat }}=\frac{\partial U(T)}{\partial T}=\frac{\partial}{\partial T} \int_{-\infty}^{+\infty} f(E, \mu, T) \cdot(E-\mu) \cdot D(E) \mathrm{d} E
$$

where $\mu$ the chemical potential, $D(E)$ is the density of states, and $f(E, \mu, T)$ is the Fermi Dirac distribution function giving by:

$$
f(E, \mu, T)=\frac{1}{1+\exp \left(\frac{E-\mu}{K_{B} T}\right)}
$$

where $K_{B}$ is the Boltzmann's constant, $T$ is the absolute temperature, $E$ is the energy of the single particle state, $\mu$ is the chemical potential and $D(E)$ is the density of states DOS. The temperature derivation in Equation (1) then acts only on $f(E, \mu, T) . S_{\text {heat }}$ behavior of can be determined once the density of electron $N$ and the chemical potential $\mu$ are known.

where:

$$
N=\int_{-\infty}^{+\infty} f(E, \mu, T) \cdot D(E) \mathrm{d} E
$$

To calculate the density of electron at each Landau level we need to know the density of states function for electrons.

Ideally, the DOS of a non-interacting $2 \mathrm{DEG}$ is given as a series of delta function [18], that is:

$$
D(E)=D_{0} \sum_{n} \delta\left(E-E_{n}\right)
$$

where $D_{0}$ is a constant which depend on devices mass, And $E_{n}=\frac{h^{2} K^{2}}{2 m}$, where $K=\frac{n \pi}{a}$, is the energy of the $n^{\text {th }}$ Landeau level. Such a DOS structure can be used to model actual materials with a very narrow distribution of energy carrier [38] [39] [40]. The delta-like density of states in Equation (4) is deformed due to scattering of electrons by impurities. As result the density of states becomes broadened.

Usually, the actual shape of the density of states of a $2 D E G$ is determined by making theoretical fits to the heat capacity data from experimental measurements. There are many form of the DOS used in literature [41] [42] [43] [44] [45] such as Lorentzian, Gaussian with a constant back ground. A derivation of the level broadening $\Gamma$ is given by Ando and Uemura [41] where they found a Lorentzian by of broadening type of broadening, are also suggested [18] [32] are a 
widely used. On Thermodynamic properties of 2 DEG systems, theoretical studies use a Gaussian Function of DOS given by:

$$
D(E)=D_{0} \sum_{n} \frac{1}{\sqrt{2 \pi} \Gamma} \exp \left(-\frac{\left(E-E_{n}\right)^{2}}{2 \Gamma^{2}}\right)
$$

where, $\Gamma$ is the broadening is taken into account by the parameter.

When the chemical potential is temperature dependent [2] [46] [47], we can calculate the derivation of the chemical potential. This will yield two terms in the specific heat capacity as giving in [48] [49] [50]. But aside from Fermi function $f(E, \mu, T)$, the specific heat capacity has also an explicit dependence on $\frac{\partial \mu}{\partial T}$.

Evaluating the temperature derivative in Equation (1) results into:

$$
S_{\text {heat }}=\int_{-\infty}^{+\infty} \frac{\partial f(E, \mu, T)}{\partial T} \cdot(E-\mu) \cdot D(E) \mathrm{d} E-\int_{-\infty}^{+\infty} f(E, \mu, T) \cdot \frac{\partial \mu}{\partial T} \cdot D(E) \mathrm{d} E
$$

Using in this expression the derivative equation of the Fermi function, we can obtain the general equation for the specific heat capacity.

\section{Quantitative Interpretation of the Temperature Dependence of Heat Capacity as Function of Chemical Potential}

In the Framework of the effective mass approximation in two-dimensional electron gas system, the Schrödinger equation in the effective mass is giving by:

$$
H \psi(x, y, z)=E \psi(x, y, z)
$$

With Hamiltonian subjected with an electrical potential, in a Cartesian system, written in this form:

$$
H=-\frac{\hbar^{2}}{2 m^{*}} \Delta+V(z)
$$

where $V(z)$ is the self-consistently calculated potential energy which includes contribution arising both from dopant charges and electrons localized in the quantum well and it is expressed as $V(z)=\frac{e}{C} \vec{A}, m^{*}$ is the effective mass, $\hbar$ is Planck's constant divided by $2 \pi$, and $E$ is the energy eigenvalue.

By computing the solution of the Schrödinger equation, we can assuming that the Landeau levels, of the $n^{\text {th }}$ energy level is $E_{n}=\frac{h^{2} K^{2}}{2 m}$. This result is clearly represented by the different states of energy for different point in the band gap (Figure 1).

Assuming a Gaussian distribution of defect states in the gap, broad distribution of the density of states was found in InAlAs devices. This result can be represented by Figure 2, which shows the presence of three peaks in the case of five states. The resolution of Schrödinger equation and density of states DOS and Fermi Dirac distribution can help us to establish the specific heat capacity equation. 


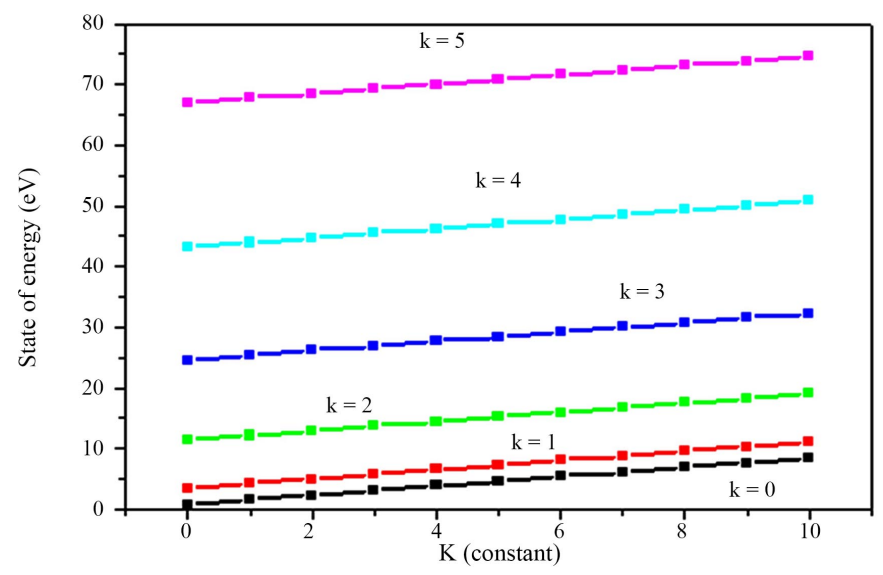

Figure 1. States of energy for different point.

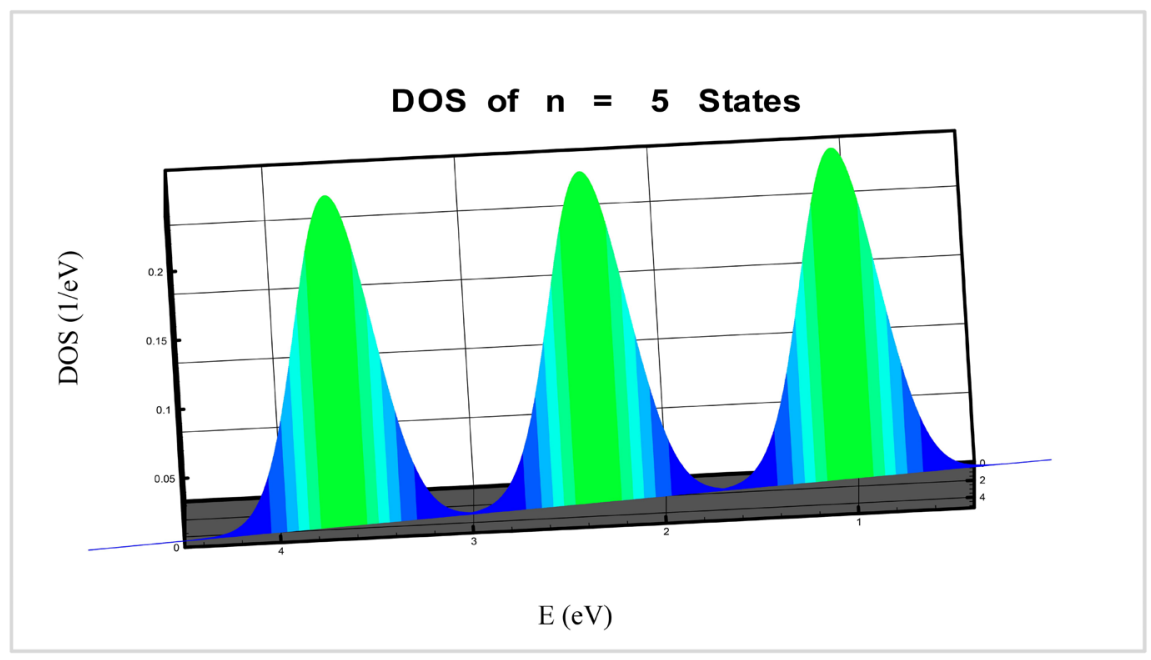

Figure 2. Density of state DOS.

The obtained distribution of density of states can now be used to calculate the temperature dependences of the heat capacity in a wide temperature range, for the case when the chemical potential $\mu$ is varied between $0 \mathrm{meV}$ to $0.7 \mathrm{meV}$ in 2 DEG semiconductor III-V, and with a fixed value of parameters $\Gamma=0.4 \mathrm{meV}$. This result was represented by Figure 3 . We note that for various $\mu$, $S_{\text {heat }}$ exhibit a single peak at the low temperature (as it is clearly shown in the insert of Figure 3). As shown in this Figure, the value of $T$ when the $S_{\text {heat }}$ is maximum, $T_{\text {peak }}$, is equal to $(12.7 \pm 1.2) \mathrm{K}$. Let's us note that the peak temperatures of the heat capacity are shifting with chemical potential $\mu$. This is expected, since we are computing the Specific heat capacity. If we computed the total heat capacity of the system, these peaks would occur at the same temperature.

$S_{\text {heat }}$ increase with the increasing of $T$, then display a sharp peak at $T_{\text {peak }}$, before the decreasing at high $T$. The maximum values occurs at a temperature very closed to that shown in this figure indicating that the observed features are likely related to Schottky behavior. This behavior happens when the heat capacity of the nuclei is comparable to that of the electron. 
While the temperature at which the peak in $S_{\text {heat }}$ appears is about $(12.7 \pm 1.2)$ $\mathrm{K}$, there is no charge of the peak temperature for a further increase of chemical potential $\mu$. Another point in support of this is the observation of the broadening of the peak become strengthen when $\mu$ increase; add the width of the peak become more large with the $\mu$ decrease.

The peak in $S_{\text {heat }}$ vs. $T$ observed at low temperature is dependent of $\mu$. Whereas the insert of Figure 3 shows that the intensity of the peak temperature $T_{c}$ strongly depends on $\mu$. The origin of the peak at very low temperature $T$ is discussed in relation with a phase transition in the electronic system.

This phenomenon is also observed in the same device but at another value of parameter $\Gamma$ equal to $0.1 \mathrm{meV}$ (Figure 4). On the plots of this figure we carried out the peak at the temperature around about $(12.85 \pm 1.25) \mathrm{K}$, which have been clarified in Ref. [51]. We would also like to show that heat capacity is affected not only by temperature, but also by chemical potential and Broadening parameter.

These results obtained at $\Gamma=0.1 \mathrm{meV}$ are in good agreement with those at $\Gamma=$ $0.4 \mathrm{meV}$, with a monotonous increase of $S_{\text {heat }}$ with the increasing of temperature. This exothermic peak proves the presence of anomaly behavior in InAlAs. Taking into account the thermodynamic properties and structural measurement, we propose that the presence of this anomaly is clarified on the basis of the idea of the Schottky anomaly.

The presence of peaks at different value of the chemical potential $\mu$, and in a wide temperature range, correspond to anomaly is an observed effect where, the specific heat capacity shows an exothermic peak. These anomalies are likely related to Schottky anomaly behavior which confirms the presence of defect in semiconductor. The shape and the sharpness of the peaks are suggestive of a phase transition in InAlAs, which we propose that they are in reality states of trapped defect [52].

Specific heat capacity for a fixed value of chemical potential $(\mu=0.5 \mathrm{meV})$ and at two different broadening parameter $\Gamma$, one equal to $0.1 \mathrm{meV}$ and the other fixed at $0.4 \mathrm{meV}$, are compared in Figure 5. This result make sure that the specific heat capacity at a fixed $\mu$ value have the same peaks at the same temperature $T=11.6 \mathrm{~K}$, for different broadening parameters $\Gamma$. Figure 6 confirms our results for another value of chemical potential $\mu$ at $0 \mathrm{meV}$. This curve shows the same behavior.

Now, we discuss the relation between specific heat capacity $S_{\text {heat }}$ vs. critical temperature $T_{c}$ of the maximum of peaks observed at low temperature in our devices, at $0.1 \mathrm{meV}$ and at $0.4 \mathrm{meV}$ parameters $\Gamma$, and for a large scale of chemical potential $\mu$ varying between $0 \mathrm{meV}$ to $0.7 \mathrm{meV}$. The relation between heat capacity and critical temperature are studied by different auteurs [53] [54].

Specific heat capacity is linear proportional to the maximum of peak temperature as shown in Figure 7 . The increasing flattening of $S_{\text {heat }}$ peak with the increasing of temperature is clearly visible. The magnitude of specific heat capacity jumps at the transition temperature $T_{c}$

The strong and non-monotonic dependence of $T_{c}$ on chemical potential $\mu$ 
(Figure 8) is a clear evidence for the fact that whichever physical effect is responsible for the peak in heat capacity, it finds its origins in a critical phenomenon, and probably a phase transition, occurring in the 2 DEG.

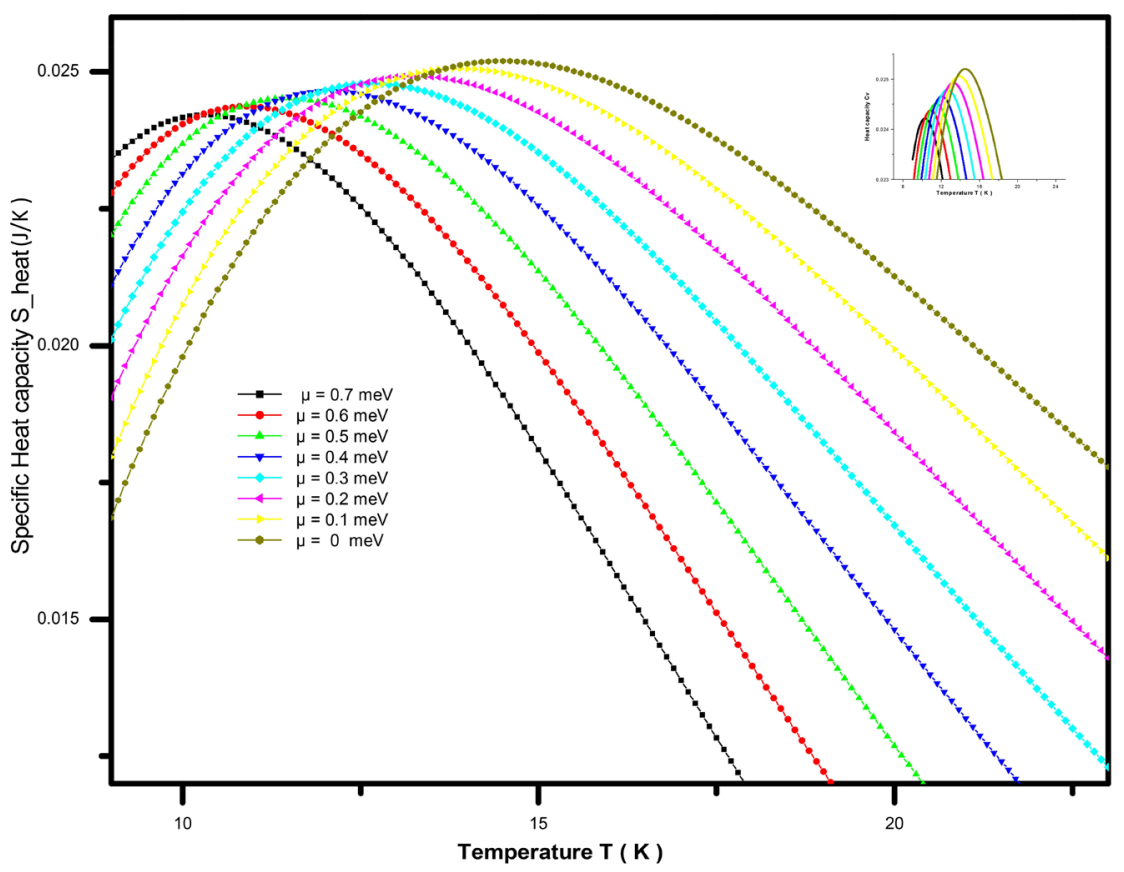

Figure 3. Temperature dependence of specific heat capacity $S_{\text {heat }}$ at temperature dependence of the InAlAs of the specific heat capacity at different chemical potential $\mu$, for a fixed Broadening parameter, $\Gamma=0.4 \mathrm{meV}$.

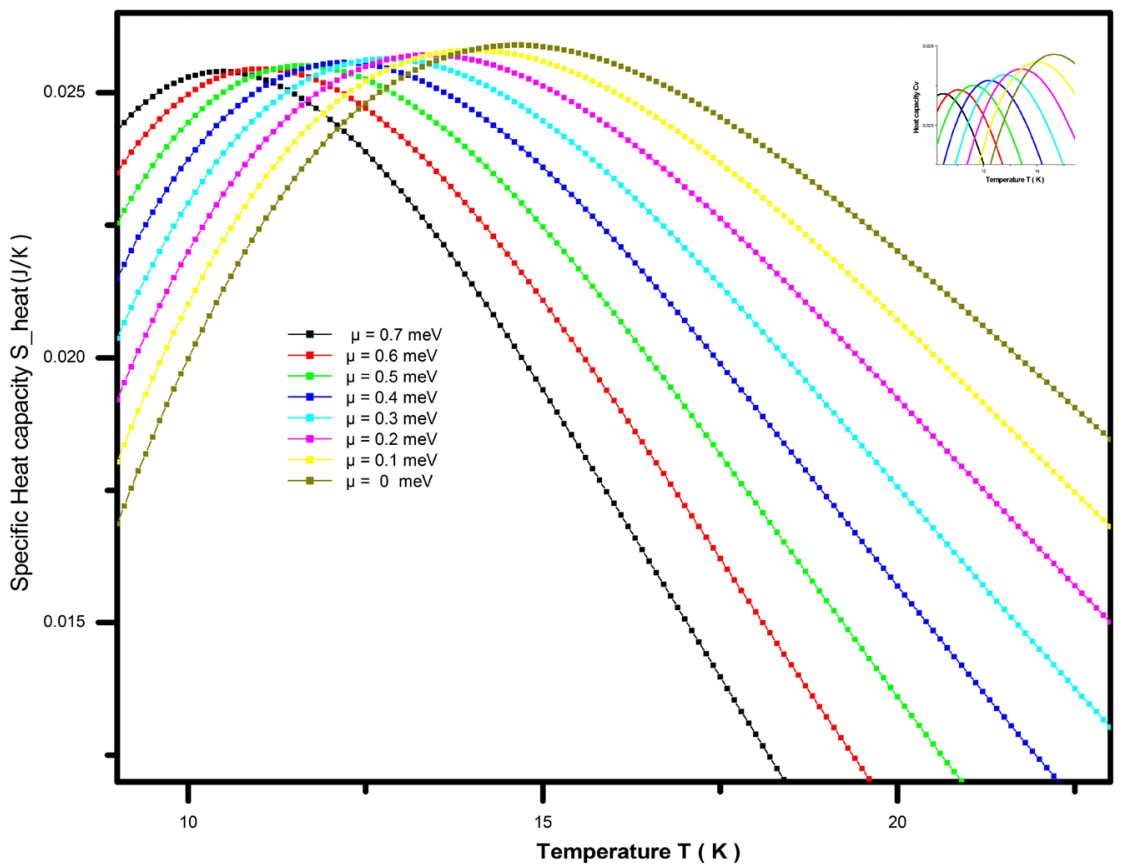

Figure 4. Temperature dependence of specific heat capacity $S_{\text {heat }}$ at temperature dependence of the InAlAs of the specific heat capacity at different chemical potential $\mu$, for a fixed Broadening parameter, $\Gamma=0.1 \mathrm{meV}$. 


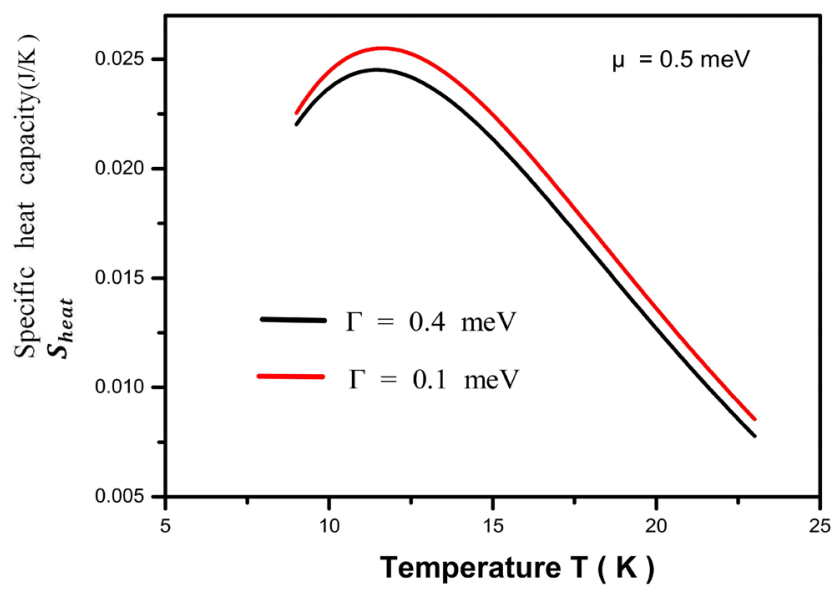

Figure 5. Specific heat capacity $S_{\text {heat }}$ at constant chemical potential $(\mu=0.5$ $\mathrm{meV}$ ) and for different Broadening parameter $\Gamma$ as a function of temperature.

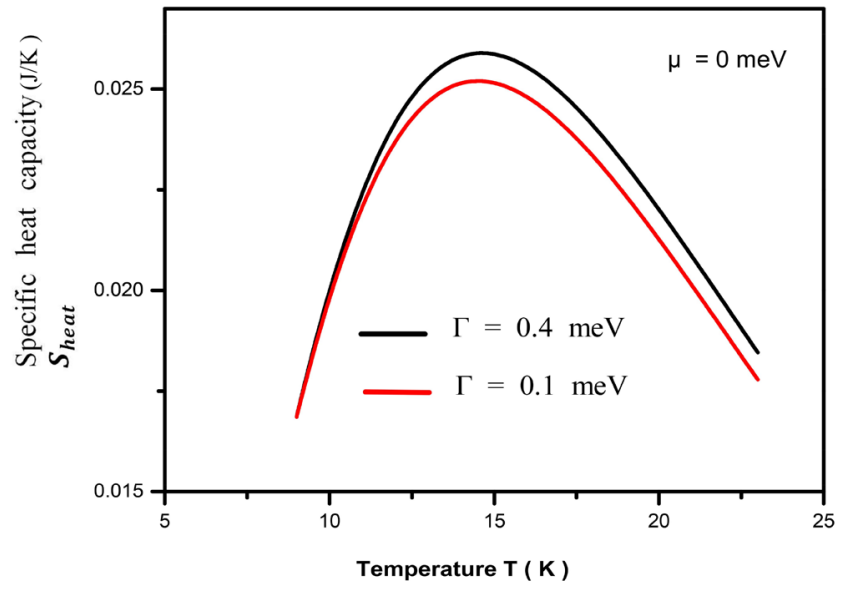

Figure 6. Specific heat capacity $S_{\text {heat }}$ at constant chemical potential $(\mu=0 \mathrm{meV})$ and for different Broadening parameter $\Gamma$ as a function of temperature.

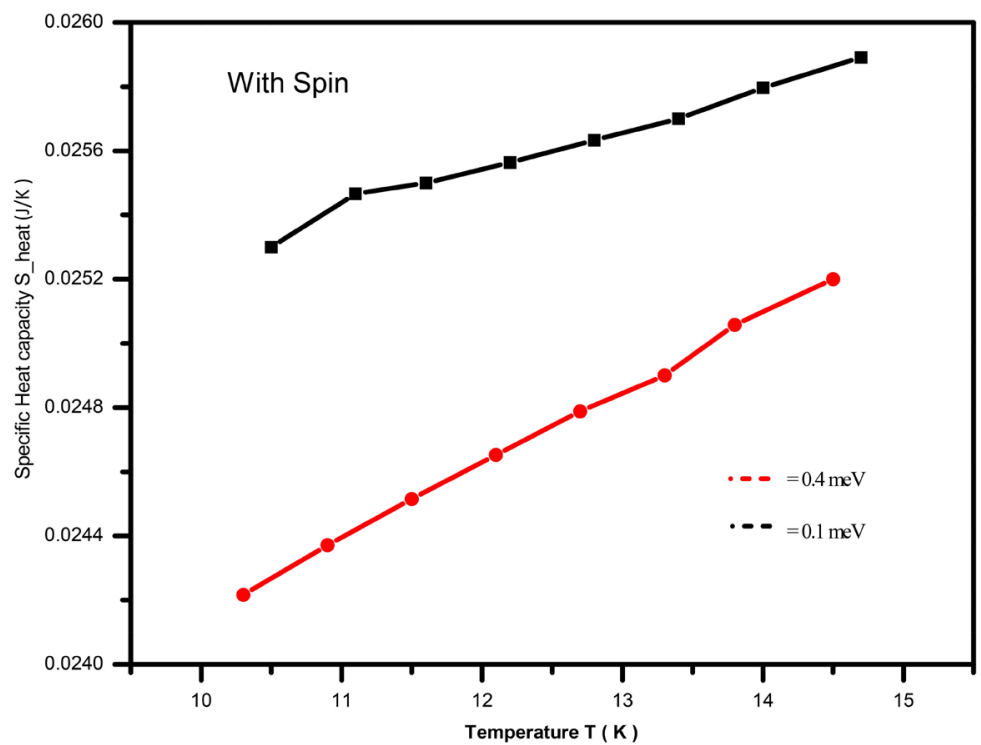

Figure 7. Specific heat capacity $S_{\text {heat }}$ peaks as a function of critical temperature $T_{c}$. 


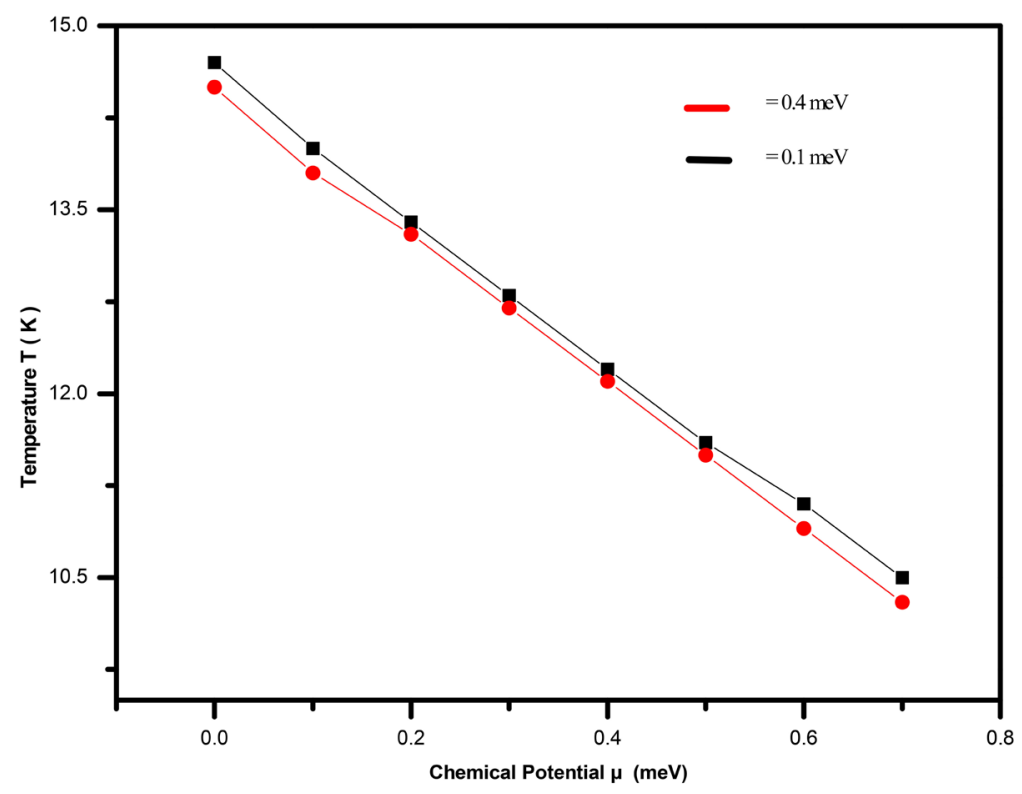

Figure 8. Critical temperature $T_{c}$ of peaks in $S_{\text {heat }}$ vs. $T$, is plotted as function of chemical potential $\mu$.

The linear decrease of critical temperature $T_{c}$ with the increase of chemical potential $\mu$ in our device is confirmed in Figure 8. This confirms that Heat capacity increase with temperature, due to the increasing number of excited degrees of freedom, requiring more energy to cause the temperature rise of peak. Specific heat capacity of critical temperature peak, also, decreases with the chemical potential $\mu$, as shown by Figure 9 .

To complete the discussion on the heat capacity, let us discuss the dependence of the heat capacity on the spin size on the InAlAs devices as a function of temperature [55]. Figure 10 shows that when the spin size is charged from 1/2 to 5/2. The position of the peak in the heat capacity, along with its height and width, is shifted to higher temperature.

Notice that with increasing of spin size the peak structure shifts to higher temperature due to the increased interaction energy that leads to a larger gap in the energy spectrum. Also, the insert of Figure 10 confirms the importance of the coupling between the 2DEG and spins. These results have the same behavior with or without spin.

\section{Conclusion}

We have studied the cardinal behavior of the specific heat capacity $S_{\text {heat }}$ of two-dimensional electron gas (2DEG) systems which are performed on InAlAs hetero-structures electron layers. Specific heat capacity has been simulated for different chemical potential and for different broadening parameter. This study exhibits remarkable peaks temperatures, which are shifting for different parameters varying in our studies. The magnitude of the specific heat capacity jumps to the critical temperature $T_{c}$ and the exponentially vanishing specific heat at low 


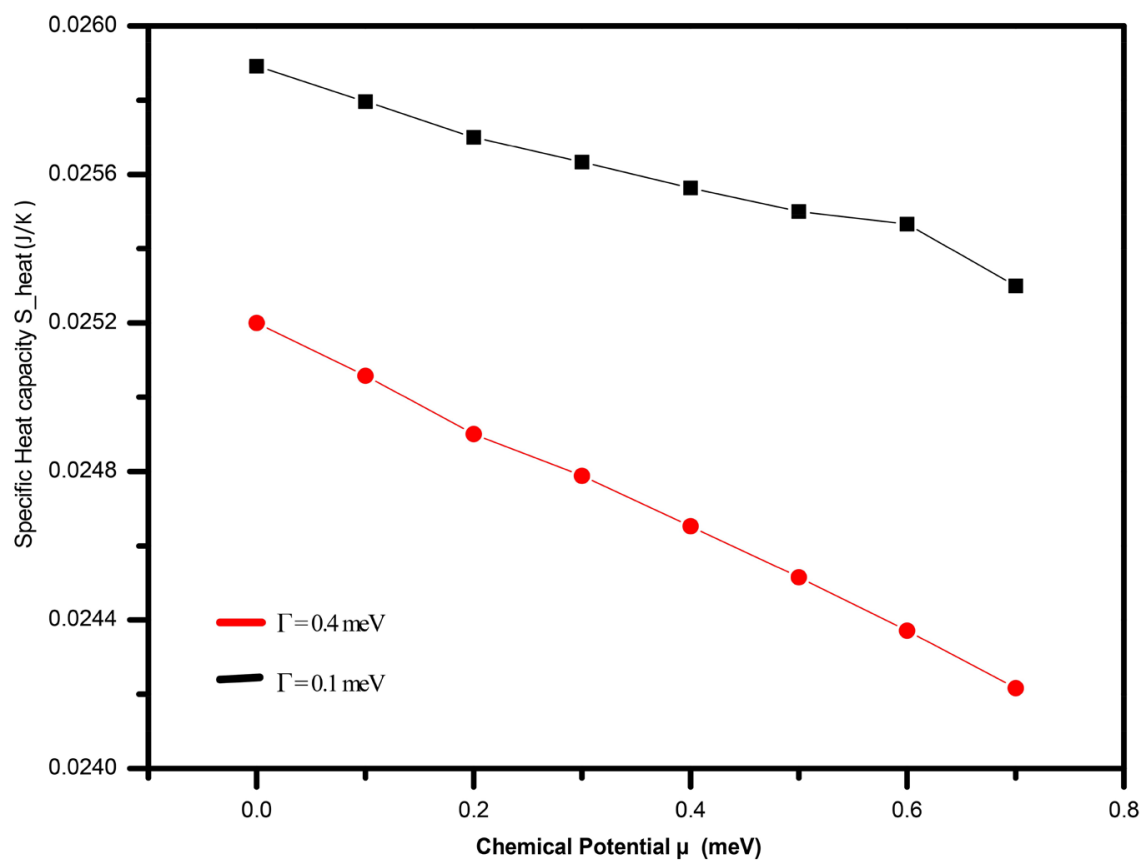

Figure 9. Specific heat capacity peaks as a function of chemical potential $\mu$ for different Broadening parameter $\Gamma$.

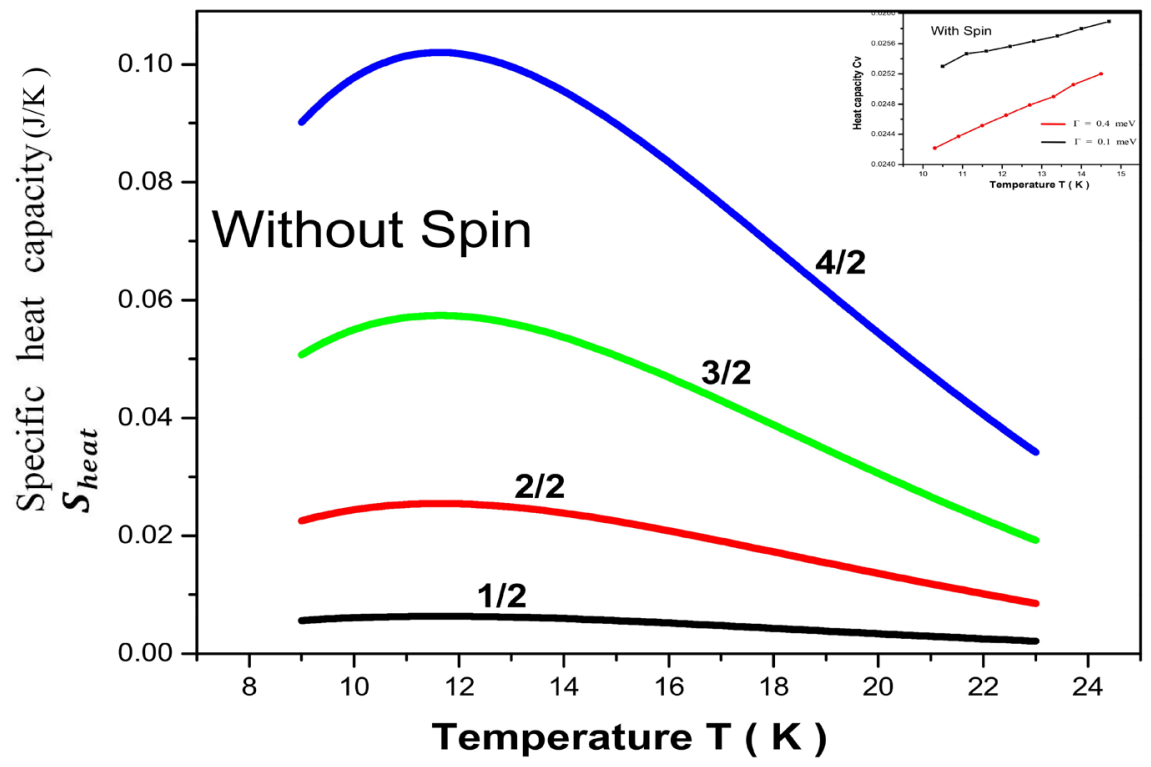

Figure 10. Specific heat capacity for $\mu=0.5 \mathrm{meV}$ and for different values of the spin varied from $1 / 2$ to $5 / 2$.

temperature, unveiling the existence of phase transition in InAlAs device, occurring the 2DEG. We suggest that this anomaly deduced from the specific heat capacity is directly related to Schottky anomaly. The study of critical temperature $T_{\mathcal{c}}$, is also made with different chemical potential $\mu$ and at $0.4 \mathrm{meV}$ and $0.1 \mathrm{meV}$ broadening parameter $\Gamma$. Our data underscore the importance of the coupling between the 2DEG and spins. These results, with spin, have the same behavior without spin. 


\section{Conflicts of Interest}

The author declares no conflicts of interest regarding the publication of this paper.

\section{References}

[1] Davies, J.H. (1998) The Physics of Low Dimensional Semiconductors: An Introduction. Cambridge University Press, Cambridge.

[2] Nizhankovskii, V.I. (2011) Thermodynamics of Two Dimensional Electron Gas in a Magnetic Field. Physics Research International, 2011, Article ID: 742158. https://doi.org/10.1155/2011/742158

[3] Sarma, S.D. and Pinczuk, A. (1997) Perspectives in Quantum Hall Effects: Novel Quantum Liquids in Low-Dimensional Semiconductor Structures. Wiley, New York.

[4] Girvin S.M. (1999) The Quantum Hall Effect: Novel Excitations and Broken Symmetries. In: Comtet, A., Jolicœur, T., Ouvry, S. and David, F., Eds., Aspects Topologiques de la Physique en Basse Dimension. Topological Aspects of Low Dimensional Systems. Les Houches-Ecole dEte de Physique Theorique, Vol. 69, Springer, Berlin, 1087.

[5] Wilde, M.A., Schwarz M.P., Heyn, C., Heitmann, D., Grundler, D., Reuter, D. and Wieck, A.D. (2006) Experimental Evidence of the Ideal de Haas-Van Alphen Effect in a Two-Dimensional System. Physical Review B, 73, Article ID: 125325. https://doi.org/10.1103/PhysRevB.73.125325

[6] Zhu, M., Usher, A., Matthews, A.J., Potts, A., Elliott, M., Herrenden-Harker, W.G., Ritchie, D.A. and Simmons, M.Y. (2003) Magnetization Measurements of HighMobility Two-Dimensional Electron Gases. Physical Review B, 67, Article ID: 155329. https://doi.org/10.1103/PhysRevB.67.155329

[7] Wang, Z.G., Zhang, W. and Zhang, P. (2009) Magnetization in Two-Dimensional Electron Gas in a Perpendicular Magnetic Field: The Roles of Edge States and Spin-Orbit Coupling. Physical Review B, 79, Article ID: 235327. https://doi.org/10.1103/PhysRevB.79.235327

[8] Dabiran, A.M., Zeller, R.J., Fang, F.F., Wright, S.L. and Stiles P.J. (1998) Electrochemical Potential Oscillations of The Two-Dimensional Electron Gas in GaAs/AlGaAs Heterostructures in High Magnetic Fields. Surface Science, 196, 712-718.

https://doi.org/10.1016/0039-6028(88)90767-4

[9] Nizhankovskii, V.I., Mokerov, V.G., Medvedev, B.K. and Shaldin, Y.V. (1986) An Investigation of the Effect of a Magnetic Field on the Chemical Potential of Electrons in Bismuth and in a $\mathrm{GaAs}-\mathrm{Al}_{\mathrm{x}} \mathrm{Ga}_{1-\mathrm{x}} \mathrm{As}$ Heterojunction. Zhurnal Eksperimentalnoi i Teoreticheskoi Fiziki, 90, 1326-1335.

[10] Firoz Islam, S.K., Naveen K. Sing and Ghosh, T.K. (2011) Thermodynamic Properties of a Magnetically Modulated Graphene Monolayer. Journal of Physics. Condensed Matter, 23, Article ID: 445502.

[11] Özdemir, B., Yarar, Z. and Özdermir, M. (2004) Variation of Chemical Potential Oscillations of a 2DEG in a Quantum Well under a Magnetic Field for Multiple Sub-Band Occupation as Function of Temperature and Level-Broadening. Turkish Journal of Physics, 28, 1-15.

[12] Tchoffo, M., Fouokeng, G.C., Fai, L.C. and Ateuafack, M.E. (2013) Thermodynamic Properties and Decoherence of a Central Electron Spin of Atom Coupled to an Anti-Ferromagnetic Spin Bath. Journal of Quantum Information Science, 3, 10-15. https://doi.org/10.4236/jqis.2013.31003 
[13] Thuy Nguyen, N.T. (2010) Quantum Dots Doped with Few Magnetic Impurities Kwantumstippen Gedopeerd Met Enkele Magnetische Onzuiverheden. University Antwerpen, Antwerpen.

[14] Al-Omari, A. (2011) Thermal Properties of Ferrimagnetic Systems. World Journal of Condensed Matter Physics, 1, 121-129. https://doi.org/10.4236/wjcmp.2011.14018

[15] Sampathkumaran, E.V., Hiroi, Z., Rayaprol, S. and Uwatoko, Y. (2004) Heat-Capacity Anomalies in the Presence of High Magnetic Fields in the Spin-Chain Compound, $\mathrm{Ca}_{3} \mathrm{Co}_{2} \mathrm{O}_{6}$. Journal of Magnetism and Magnetic Material, 284, L7-L11. https://doi.org/10.1016/j.jmmm.2004.07.028

[16] Mahdavifar, S. and Akbari, A. (2008) Heat Capacity of Schottky Type in Low-Dimensional Spin Systems. Journal of Physics: Condensed Matter, 20, Article ID: 215213. https://doi.org/10.1088/0953-8984/20/21/215213

[17] Eisenstein, J.P., Stormer, H.L., Narayanamurti, V., Cho, A.Y., Gossard, A.C. and Tu, C.W. (1985) Density of States and de Haas-Van Alphen Effect in Two-Dimensional Electron Systems. Physical Review Letters, 55, 875. https://doi.org/10.1103/PhysRevLett.55.875

[18] Ando, T., Fowler, A.B. and Stern, F. (1982) Electronic Properties of Two-Dimensional Systems. Reviews of Modern Physics, 54, 437. https://doi.org/10.1103/RevModPhys.54.437

[19] Ramos, A.C.A., Alves T.F.A., Farias, G.A., Costa Filho, R.N. and Almeida, N.S. (2009) 2DEG in the Presence of Tilted Magnetic Field at Finite Temperature. Physica E: Low-Dimensional Systems and Nanostructures, 41, 1267-1271. https://doi.org/10.1016/j.physe.2009.02.011

[20] Xie, X.C., Li, Q.P. and Das Sarma, S. (1990) Density of States and Thermodynamic Properties of a Two-Dimensional Electron Gas in a Strong External Magnetic Field. Physical Review B, 42, 7132. https://doi.org/10.1103/PhysRevB.42.7132

[21] Meinel, I., Grundler, D., Heitmann, D., Manolescu, A., Gudmundsson, V., Wegscheider, W. and Bichler, M. (2001) Enhanced Magnetization at Integer Quantum Hall States. Physical Review B, 64, Article ID: 121306. https://doi.org/10.1103/PhysRevB.64.121306

[22] Smith, T.P., Goldberg, B.B., Stiles, P.J. and Heiblum, M. (1985) Direct Measurement of the Density of States of a Two-Dimensional Electron Gas. Physical Review B, 32, 2696. https://doi.org/10.1103/PhysRevB.32.2696

[23] Das Sarma, S. and Xie, X.C. (1988) Strong-Field Density of States in Weakly Disordered Two-Dimensional Electron Systems. Physical Review Letters, 61, 738-741. https://doi.org/10.1103/PhysRevLett.61.738

[24] Gopal, E.S.R. (1966) Specific Heats at Low Temperatures. In: Mendelssohn, K. and Timmerhaus, K.D., Eds., Heywood Books, Plenum Press, London, 63.

[25] Melinte, S., Grivei, E., Beuken, J.M., Mariage, G., Malcorps, L., Gustin, C., Bayot, V. and Shayegan, M. (2000) The Effect of Zeeman Energy on Heat Capacity of GaAs/AlGaAs Heterostructures near $v=1$. Physica E: Low-Dimensional Systems and Nanostructures, 6, 52-55. https://doi.org/10.1016/S1386-9477(99)00054-5

[26] Bayot, V., Grivei, E., Melinte, S., Santos, M.B. and Shayegan, M. (2008) Giant Low Temperaure Heat capacity of GaAs Quantum Wells near Landau Level Filline. Condensed Matter, 1, Article ID: 9603024.

[27] Baker, P.J., Giblin, S.R., Pratt, F.L., Liu, R.H., Wu, G., Chen, X.H., Pitcher, M.J., Parker, D.R., Clarke, S.J. and Blundell, S.J. (2008) Heat Capacity Measurements on 
FeAs Based Compounds: A Thermodynamic Probe of Electronic and Magnetic States. Condensed Matter, 1, 2494.

[28] He, C., Zheng, H., Mitchell, J.F., Foo, M.L., Cava, R.J. and Leighton, C. (2009) Low Temperature Schottky Anomalies in the Specific Heat of $\mathrm{LaCoO}_{3}$ : Defect-Stabilized Finite Spin States. Applied Physics Letters, 94, Article ID: 102514. https://doi.org/10.1063/1.3098374

[29] Xie, X.C., Li, Q.P. and Sarma, S.D. (1990) Density of States and Thermodynamic Properties of a Two-Dimensional Electron Gas in a Strong External Magnetic Field. Physical Review B, 42, 7132-7147. https://doi.org/10.1103/PhysRevB.42.7132

[30] Viallogonzalo, C. and Gammag, R. (2010) The Intrinsic Features of the Specific Heat at Half Filled Landau Levels of Two Dimensional Electron Systems. Journal Low Temp Physics, 2, 3802.

[31] Kuzmenko, N.K. and Mikhajlov, V.M. (2008) The Canonical Heat Capacity of Normal Mesoscopic Fermion Systems: The Temperature Evolution and Particle Number Oscillations. Condensed Matter Statistical Mechanics, 1, 2078.

[32] Gornik, E., Lassnig, R., Strasser, G., Störner, H.L., Gossard, A.C. and Wiegmann, W. (1985) Specific Heat of Two-Dimensional Electrons in GaAs-GaAlAs Multilayers. Physical Review Letters, 54, 1820-1823. https://doi.org/10.1103/PhysRevLett.54.1820

[33] Cerez, A., Henry, M. and Varret, F. (1980) Isotropy of the Lamb Mossbauer Factor in Ferrous Fluosillicate Single Crystals. Journal de Physique Letteres, 41, L157-L159.

[34] Gai, H.F., Wang, J. and Tian, Q. (2007) Modified Debye Model Parameters of Metals Applicable for Broadband Calculations. Applied Optics, 46, 2229-2233. https://doi.org/10.1364/AO.46.002229

[35] Moroyoqui-Estrella, G., Rodriguez-Mijangos, R., Perez-Salas, R. and Rodriguez, A. (2013) Thermal Properties of High Order Crystalline Dielectric Mixtures. Revista Mexicana de Fisica, 59, 16-19.

[36] Sahling, S., Lorenzo, J.E., Remenyi, G. and Katkov, V.L. (2019) Low-Temperature Heat Capacity of Sr_2Ca_\{12\}Cu_\{24\}O_\{41\}. Journal of Low Temperature Physics, 194, 142-152.

[37] Bozdogana, A.E. and Bozdogan, I.S. (2019) New Equations for Lattice and Electronic Heat Capacities, Enthalpies, and Entropies of Solids: Application to Diamond. Acta Physica Polonica A, 135, 674-677. https://doi.org/10.12693/APhysPolA.135.674

[38] Han, Y., Gu, P.F., Liu, F.J., Liu, W., Wang, L., Zhang, X. and Lin, Y.C. (2019) Analysis between Subgap Density of State and NBIS of Top Gate a IGZO TFTs. BOE Technology, 9, 50-76.

[39] Akinlami1, J.O., Omeike M.O. and Akindiilete, A.J. (2019) Electronic, Structural and Paramagnetic Properties of Magnesium Telluride. SPQEO, 22, 5-10.

[40] Mostefai, A., Berrah, S. and Abid, H. (2018) Electronics Properties of Monoclinic HfO2. Journal of Nano- and Electronic Physics, 10, Article ID: 06026. https://doi.org/10.21272/jnep.10(6).06026

[41] Ando, T. and Uemura, Y.J. (1974) Theory of Quantum Transport in a Two-Dimensional Electron System under Magnetic Fields. I. Characteristics of Level Broadening and Transport under Strong Fields. Journal of the Physical Society of Japan, 36, 959-957. https://doi.org/10.1143/JPSJ.36.959

[42] Wang, S.H., Wan, C.P., Heng, Y.X. and Ao, J.-P. (2019) Effect of Grinding-Induced Stress on Interface State Density of $\mathrm{SiC} / \mathrm{SiO}_{2}$. Materials Science Forum, 954, 121-125. 
https://doi.org/10.4028/www.scientific.net/MSF.954.121

[43] Ahmadabadi, H.N. and Ghafouri-Khosrowshahi, A. (2018) Effect of Thermal Dependency of Diameter on Density of States for Zigzag Carbon Nano-Tubes. Journal of Nano Research, 55, 1-10. https://doi.org/10.4028/www.scientific.net/JNanoR.55.1

[44] Xu, H.Y., Wan, C.P. and Ao, J.-P. (2019) The Correlation between the Reduction of Interface State Density at the $\mathrm{SiO}_{2} / \mathrm{SiC}$ Interface and the NO Post-Oxide-Annealing Conditions. Materials Science Forum, 954, 104-108. https://doi.org/10.4028/www.scientific.net/MSF.954.104

[45] Swain, R., Sahu, S. and Rout, G.C. (2018) Tight-Binding Theoretical Study of the Tunneling Conductance in Ferromagnetically Ordered Graphene-on-Substrate. Journal of Superconductivity and Novel Magnetism, 31, 2519-2528. https://doi.org/10.1007/s10948-017-4502-x

[46] Xie, X.C., Li, Q.P. and Das Sarma, S. (1990) Density of States and Thermodynamic Properties of a Two-Dimensional Electron Gas in a Strong External Magnetic Field. Physics Review B, 142, 7132-7147. https://doi.org/10.1103/PhysRevB.42.7132

[47] Özdemir, B., Yarar, Z. and Özdemir, M. (2004) Variation of Chemical Potential Oscillations of a 2DEG in a Quantum Well Under a Magnetic Field for Multiple Sub-Band Occupation as Function of Temperature and Level Boardening. Turkish Journal of Physics, 28, 1-15.

[48] Firiz Islam, S.K. Singh, N.K. and Ghosh, T.K. (2011) Thermodynamic Properties of Magnetically Modulated Graphene. Condensed Matter Mesoscale and Nanoscale Physics, 2, 3060.

[49] Zawadzki, W. and Lassning, R. (1984) Specific Heat and Magneto-Thermal Oscillations of Two-Dimensional Electron Gas in a Magnetic Field. Solid State Communications, 50, 537-539. https://doi.org/10.1016/0038-1098(84)90324-7

[50] Zawadzki, W. and Lassning, R. (1984) Magnetization, Specific Heat, Magneto-Thermal Effect and Thermoelectric Power of Two-Dimensional Electron Gas in a Quantizing Magnetic Field. Surface Science, 142, 225-235. https://doi.org/10.1016/0039-6028(84)90312-1

[51] Zhao, Y. and Franco, F. (2014) Ring Polymers with Topological Constraints. Condensed Matter, 1402.

[52] Bouzgarrou, S., Ben Salem, M.M., Kalboussi, A. and Souifi, A. (2013) Experimental and Theoretical Study of Parasitic Effects in InAlAs/InGaAs/InP HEMT's. American Journal of Physics and Application, 1, 18-24. https://doi.org/10.11648/j.ajpa.20130101.14

[53] Bianconi, A., Agrestini, S., Campi, G., Filippi, M. and Saini, N.L. (2005) Common Features in High $T_{\mathrm{c}}$ Cuprates and Diborides. Current Applied Physics, 5, 254-258. https://doi.org/10.1016/j.cap.2004.02.010

[54] Grivei, E., Beuken, J.M., Mariage, G., Bayot, V. and Shayegan, M. (1998) Heat Capacity of a 2DEG. Physica B: Condensed Matter, 256-258, 90-96. https://doi.org/10.1016/S0921-4526(98)00568-7

[55] Park, J.H. Lee, S., Lee, H.S., Kim, S.K., Park, K.-S. and Yoon, S.-Y. (2018) Correlation between Spin Density and $\mathrm{V}_{\text {th }}$ Instability of IGZO Thin-Film Transistors. Current Applied Physics, 18, 1447-1450. https://doi.org/10.1016/j.cap.2018.08.016 